\title{
Differential Reactions on Mature Green and Ripe Chili Fruit Infected by Three Colletotrichum spp.
}

Orarat Mongkolporn, Department of Horticulture and Center for Agricultural Biotechnology, Kasetsart University, Kamphaeng Saen Campus, Nakhon Pathom 73140 Thailand/Center for Agricultural Biotechnology (AGBIO/PERDO-CHE); Paweena Montri, Center for Agricultural Biotechnology, Kasetsart University/Center for Agricultural Biotechnology (AG-BIO/PERDO-CHE); Thunyawan Supakaew, Plant Breeding Program, Faculty of Agriculture at Kamphaeng Saen, Kasetsart University, Kamphaeng Saen Campus, Nakhon Pathom 73140 Thailand; and Paul W. J. Taylor, BioMarka/Center for Plant Health, Melbourne School of Land and Environment, The University of Melbourne, Victoria 3010 Australia

\begin{abstract}
Mongkolporn, O., Montri, P., Supakaew, T., and Taylor, P. W. J. 2010. Differential reactions on mature green and ripe chili fruit infected by three Colletotrichum spp. Plant Dis. 94:306-310.

Differential reactions on mature green and ripe chili fruit of 10 genotypes from four cultivated Capsicum spp. (i.e., Capsicum апnиum, C. baccatum, C. chinense, and $C$. frutescens) were investigated after being inoculated with 33 isolates of Colletotrichum capsici, C. gloeosporioides, and $C$. acutatum originating from Thailand. The inoculation was performed using an injection method. Differential reactions, based on qualitative host reactions (i.e., lesion development versus no infection) grouped the Colletotrichum isolates into different pathotypes. C. capsici was grouped into three pathotypes based on differential reactions on ripe fruit stage of two Capsicum chinense genotypes (PBC932 and C04714) and two pathotypes based on differential reactions on mature green fruit stage of C04714. Colletotrichum gloeosporioides was grouped into five and six pathotypes on their reactions in ripe and green fruit maturity stages, respectively. C04714 and two Capsicum annuum genotypes (Jinda and Bangchang) acted as differential hosts with ripe fruit, whereas $\mathrm{C} 04714$ with all $C$. annuum and $C$. frutescens genotypes acted as differential hosts with green fruit. No pathotype of Colletotrichum acutatum was identified on ripe fruit but three pathotypes were identified on green fruit based on differential reactions in two Capsicum baccatum genotypes (PBC80 and PBC81).
\end{abstract}

Anthracnose is a major disease causing fruit rot in chili (Capsicum spp.). The disease causes significant yield losses in the tropics and subtropics worldwide. Estimated values of annual production losses of US\$100 million and 1.33 million were reported in Korea (7) and India (10), respectively. Anthracnose of Capsicum spp. is caused by a complex of Colletotrichum spp., with three main species (Colletotrichum capsici, C. gloeosporioides, and $C$. acutatum) having been reported as key pathogens, particularly in Asia (5,8,10-12).

Pathotypes of $C$. capsici identified based on qualitative differential host reactions (i.e., the ability or inability to cause disease in specific host genotypes) were first reported by Montri et al. (5). Eleven $C$. capsici isolates were grouped into three

Corresponding author: P. W. J. Taylor

E-mail: paulwjt@unimelb.edu.au

Current address of P. Montri: Plant Protection, Royal Project Foundation, Chiang Mai 50200 Thailand.

Accepted for publication 30 October 2009.

doi:10.1094/PDIS-94-3-0306

(C) 2010 The American Phytopathological Society pathotypes based on differential reactions on ripe fruit of nine chili genotypes. Also, a disease severity scale, with scores from 0 to 9, was established based on percent lesion size relative to overall fruit size, with 0 representing no lesion development and 9 when the lesion covered $>25 \%$ of the fruit surface. The disease scale developed by Montri et al. (5) provided an efficient and accurate measurement of disease severity, which has been well applied to breeding programs to assess anthracnose resistance $(3,4)$. Assessment of resistance to anthracnose significantly corresponded with differential host reactions in the pathotype study. According to the inheritance studies conducted by Pakdeevaraporn et al. (6) and Mahasuk et al. (3), the resistant phenotype had a disease score of 0 while the susceptible phenotype had disease scores ranging from 1 to 9 . More interestingly, Mahasuk et al. $(3,4)$ suggested that two different genes controlled resistance at different fruit maturity stages (i.e., "mature green" and "ripe").

The indication of distinct genes responsible for resistance at different fruit maturity stages will have an impact on pathotype identification when inoculating chili genotypes at different fruit stages. Therefore, the aim of this study was to investigate differential reactions in mature green and ripe fruit of 10 chili genotypes, inoculated with the three important Colletotrichum spp. causing anthracnose of chili (i.e., $C$. capsici, C. gloeosporioides, and C. acutatum).

\section{MATERIALS AND METHODS}

Colletotrichum spp. collection and taxonomy verification. Thirty-three isolates of Colletotrichum were recovered from chili fruit infected with anthracnose in Thailand. All isolates were identified by morphological characteristics such as spore shape and size, and growth rate of cultures on potato dextrose agar (PDA; 9). Twelve isolates were identified as C. capsici (5) without further characterization. In all, 11 isolates were identified as C. gloeosporioides and 10 as C. acutatum, with their identities verified using speciesspecific primers for C. gloeosporioides and C. acutatum as described by Kim et al. (2). Isolate names and their origins are listed in Table 1. Isolations and establishment of single-spore cultures were carried out according to the procedures of Photita et al. (9) and Than et al. (11).

Colletotrichum cultures and inoculum preparation. Colletotrichum cultures derived from single conidia were grown on PDA (Difco, Becton, Dickinson and Company, Sparks, MD). Conidial suspensions of each Colletotrichum isolate were prepared to a concentration of approximately $10^{6}$ conidia/ml following the protocol of Montri et al. (5).

Chili genotypes. Ten chili genotypes of Capsicum annuum L., C. baccatum L., $C$. chinense Jacq., and $C$. frutescens L. (Table 2) were inoculated with the 33 Colletotrichum isolates.

Pathogenicity study. Fruit were harvested at the mature green stage ( 35 to 45 days after flowering, depending on genotype) and ripe stage (40 to 50 days). Five detached mature green fruit and five ripe fruit of each chili genotype were inoculated with each Colletotrichum isolate using an injection method $(1,5)$. Each fruit was injected once in the center of the fruit. Each injection contained approximately 1,000 spores. 
Anthracnose symptoms at the inoculation site were evaluated at 9 days after inoculation (DAI) based on percent lesion size (lesion size as a proportion to fruit size; 5). Mean percent lesion size was then converted to 0 -to- 9 disease severity scores, where $0=$ no lesion, $1=$ lesion size covering 1 to $2 \%$ of fruit surface, $3=>2$ to $5 \%$ lesion size, $5=>5$ to $15 \%$ lesion size, $7=$ $>15$ to $25 \%$ lesion size, and $9=$ lesions $>25 \%$ (5). All experiments were repeated and gave similar results.

Pathotypes were identified where qualitative differences in lesion development (0 versus 1-to-9 scores) occurred for isolates on a specific chili genotype at the same fruit maturity stage. Pathotype designations for each Colletotrichum spp. are as follows: PCc for Colletotrichum capsici, $\mathrm{PCg}$ for C. gloeosporioides, and PCa for C. acutatum. Suffixes $-\mathrm{R}$ and $-\mathrm{G}$ designate pathotypes identified based on reactions of ripe and mature green fruit, respectively.

\section{RESULTS}

Differential reactions of chili genotypes to $C$. capsici infection. Ripe fruit stage. Fruit of 10 Capsicum genotypes at ripe stage exhibited a range of host reactions following inoculation with the $12 \mathrm{C}$. capsici isolates. None of the three Capsi-

Table 1. Single-spore isolates $(n=33)$ of Colletotrichum capsici, C. gloeosporioides, and $C$. acutatum and their origins in Thailand

\begin{tabular}{ll}
\hline Colletotrichum spp. & \multicolumn{1}{c}{ Origin } \\
\hline C. capsici & \\
F4-2C & Kanchana Buri \\
F4-5A & Kanchana Buri \\
F7-3A & Nakhon Pathom \\
F8-1A & Suphan Buri \\
F8-2A & Suphan Buri \\
F8-3A & Suphan Buri \\
F8-3B & Suphan Buri \\
F8-4C & Suphan Buri \\
F8-5A & Suphan Buri \\
F8-5B & Suphan Buri \\
SKP16 & Chiang Mai \\
158ci & Unknown \\
C. gloeosporioides & \\
F1-3A & Ratchaburi \\
F1-3C & Ratchaburi \\
F4-1B & Kanchana Buri \\
F4-1C & Kanchana Buri \\
F5-1A & Kanchana Buri \\
F5-2D & Kanchana Buri \\
F5-4A & Kanchana Buri \\
F7-1B & Nakhon Pathom \\
F7-3B & Nakhon Pathom \\
F7-4A & Nakhon Pathom \\
DOA & Nakhon Si Thammarat \\
C. acutatum & \\
211 & Chiang Mai \\
311 & Chiang Mai \\
313 & Chiang Mai \\
314 & Chiang Mai \\
316 & Chiang Mai \\
322 & Chiang Mai \\
MJ3 & Chiang Mai \\
MJ5 & Chiang Mai \\
MJ7 & Chiang Mai \\
MJ8 & Chiang Mai \\
\hline & \\
\hline
\end{tabular}

cum baccatum genotypes developed measurable lesions, whereas all C. frutescens and $C$. annuum genotypes were susceptible to all Colletotrichum capsici isolates, receiving disease scores ranging from 5 to 9 (Table 3). Capsicum frutescens cvs. Kheenoo Suan and Karen and C. annuum line 83-168 were the most susceptible genotypes, with average scores of 9. C. chinense genotypes C04714 and PBC932 developed lesions following inoculation with some isolates but not others. Three pathotypes were identified (Table 3) based on the three differential reactions expressed by $\mathrm{C} 04714$ and PBC932 as follows: PCc1-R, six C. capsici isolates that incited lesions in both $\mathrm{C} 04714$ and PBC932; PCc2-R, four isolates that incited lesions only on C04714; and PCc3-R, two isolates that did not incite lesions on either C04714 or PBC932.

Mature green fruit stage. Host reactions of mature green fruit were different from those of the ripe fruit with the same Colletotrichum capsici isolates. Green fruit of four chili genotypes, including the three Capsicum baccatum lines and $C$. chinense line PBC932, were resistant to all $12 \mathrm{Col}$ letotrichum capsici isolates (Table 4). All Capsicum annuum and C. frutescens genotypes at the green fruit stage were susceptible to all the Colletotrichum capsici isolates with disease scores from 3 to 9 . Similar to the ripe fruit stage, mature green fruit of Capsicum annuum line 83168 and $C$. frutescens cvs. Kheenoo Suan and Karen were very susceptible to Colletotrichum capsici, with average scores of 9. Reactions of Bangchang, Jinda, and C04714 were more variable, with average scores of 8,6 , and 5 , respectively. Only C04714 gave differential reactions because isolates F7-3A and SKP16 did not incite lesions in this genotype and were classified as PCc2-G (Table 4). The remainder of the $C$. capsici isolates were classified as PCc1-G.

Differential reactions of chili genotypes to $C$. gloeosporioides infection. Ripe fruit stage. All 11 C. gloeosporioides isolates failed to incite lesions in ripe fruit of the three Capsicum baccatum lines and the $C$. chinense line PBC932. Among the
C. annuum genotypes, 83-168 was the most severely affected by Colletotrichum gloeosporioides isolates, with an average score of 9 (Table 5). Both Capsicum frutescens cvs. Kheenoo Suan and Karen were also severely affected, with average scores of 9. C. chinense line C04714 and $C$. annuum cvs. Bangchang and Jinda reacted differentially to the Colletotrichum gloeosporioides isolates. Considering only the differential reactions of C04714, Bangchang, and Jinda, five pathotypes were identified (Table 5). PCg1-R group contained five isolates that incited lesions in C04714, Bangchang, and Jinda. PCg2-R contained two isolates, F7-4A and F7-1B, that caused lesions in $\mathrm{C} 04714$ and Bangchang. PCg3-R, PCg4-R, and PCg5-R differentiated by reactions of chili genotypes C04714, Jinda, and Bangchang.

Mature green fruit stage. Reactions of mature green fruit were more varied than those on ripe fruit of the same genotypes when inoculated with the same $11 C$. gloeosporioides isolates. Specific chili genotypes that were not infected at mature green fruit stage by $C$. gloeosporioides were the same as those not infected at the ripe fruit stage (Table 6). However, the infected chili genotypes at the mature green stage showed higher variations in infection, and six pathotypes were identified (Table 6). The three most susceptible chili genotypes, Kheenoo Suan, Karen, and 83-168, were also severely infected by most C. gloeosporioides isolates; however, with slightly lower mean score of 7 . The other three chili genotypes, C04714, Bangchang, and Jinda, were only slightly infected by $C$. gloeosporioides, with average scores of 1 .

Differential reactions of chili genotypes to $C$. acutatum infection. Ripe fruit stage. $C$. acutatum isolates were the most aggressive among the three Colletotrichum spp., inciting lesion development in all genotypes of Capsicum annuum, C. frutescens, and $C$. chinense as well as line PBC1422 of $C$. baccatum (Table 7). $C$. baccatum lines $\mathrm{PBC} 80$ and $\mathrm{PBC} 81$ were highly resistant to all Colletotrichum acutatum isolates tested. PBC1422 expressed intermediate levels of resistance to all $C$.

Table 2. Ten chili genotypes of four Capsicum spp. assessed for differential reactions to Colletotrichum spp.

\begin{tabular}{lllcl}
\hline No. & Capsicum sp. & Genotype & $\begin{array}{c}\text { Code for } \\
\text { this study }\end{array}$ & \multicolumn{1}{c}{ Origin and description $^{\mathbf{a}}$} \\
\hline 1 & Capsicum annuum & Bangchang & BC & Thai elite cultivar \\
2 & & Jinda & JD & Thai elite cultivar \\
3 & & $83-168$ & 83 & AVRDC line \\
4 & C. baccatum & PBC80 & 80 & AVRDC anthracnose-resistant line \\
5 & & PBC81 & 81 & AVRDC anthracnose-resistant line \\
6 & & PBC1422 & 1422 & AVRDC line \\
7 & C. chinense & PBC932 & 932 & AVRDC anthracnose-resistant line \\
8 & & C04714 & 4714 & AVRDC line \\
9 & C. frutescens & Kheenoo Suan & KN & Thai elite cultivar \\
10 & & Karen & KR & Thai local variety \\
\hline
\end{tabular}

a AVRDC $=$ The World Vegetable Center, Shanhua, Tainan, Taiwan 
acutatum isolates, with an average score of 5. Kheenoo Suan, Karen, 83-168, and PBC932 were very susceptible to $C$. $a c u$ tatum, with all genotypes receiving scores of 9 for all isolates. Ripe fruit of the 10 chili genotypes tested did not exhibit differential reactions to any of the $C$. $a c u$ tatum isolates; therefore, no pathotype was identified (Table 7).

Mature green fruit stage. Capsicum baccatum genotypes PBC80 and PBC81, found to be resistant to all Colletotrichum acutatum isolates at the ripe fruit stage, were moderately susceptible at the mature green fruit stage to six of the $C$. acutatum isolates (Table 8). The other eight chili genotypes were susceptible to all $C$. $a c u$ tatum isolates, with severity levels similar to those at the ripe fruit stage. Three pathotypes were identified based on differential reactions in $\mathrm{PBC} 80$ and $\mathrm{PBC} 81$ in mature green fruit and designated PCa1-G, PCa2$\mathrm{G}$, and PCa3-G (Table 8). PCa1-G contained five isolates that incited lesions in both PBC80 and PBC81. PCa2-G included only isolate 313 , which incited lesions in
PBC81 but not PBC80. PCa3-G contained four isolates that failed to incite lesions in either PBC80 or PBC81.

In summary (Table 9), three pathotypes of $C$. capsici were identified at the ripe fruit stage based on differential reactions in Capsicum chinense lines PBC932 and C04714 and two pathotypes at the mature green fruit stage based on differential reactions in C04714. Colletotrichum gloeosporioides could be grouped into five pathotypes at the ripe fruit stage based on differential reactions in Capsicum chinense

Table 3. Anthracnose severity scores on a 0-to- 9 scale $^{\mathrm{a}}$ and pathotypes of 12 Colletotrichum capsici isolates at the ripe fruit stage of 10 chili genotypes

\begin{tabular}{|c|c|c|c|c|c|c|c|c|c|c|c|c|}
\hline \multirow[b]{2}{*}{ Isolate } & \multicolumn{3}{|c|}{ Capsicum baccatum } & \multicolumn{2}{|c|}{ C. chinense } & \multicolumn{3}{|c|}{ C. annuum } & \multicolumn{2}{|c|}{ C. frutescens } & \multirow[b]{2}{*}{ Mean } & \multirow[b]{2}{*}{ Pathotype } \\
\hline & 80 & 81 & 1422 & 932 & 4714 & JD & BC & 83 & $\mathbf{K N}$ & KR & & \\
\hline F4-2C & 0 & 0 & 0 & 0 & 5 & 9 & 5 & 9 & 9 & 9 & 5 & PCc2-R \\
\hline F4-5A & 0 & 0 & 0 & 0 & 9 & 9 & 9 & 9 & 9 & 9 & 5 & PCc2-R \\
\hline F7-3A & 0 & 0 & 0 & 0 & 0 & 7 & 9 & 9 & 9 & 7 & 4 & PCc3-R \\
\hline F8-1A & 0 & 0 & 0 & 9 & 9 & 9 & 9 & 9 & 9 & 9 & 6 & PCc1-R \\
\hline F8-2A & 0 & 0 & 0 & 7 & 7 & 9 & 9 & 9 & 9 & 9 & 6 & PCc1-R \\
\hline F8-3A & 0 & 0 & 0 & 9 & 7 & 7 & 9 & 9 & 9 & 9 & 6 & PCc1-R \\
\hline F8-3B & 0 & 0 & 0 & 7 & 9 & 9 & 9 & 9 & 9 & 9 & 6 & PCc1-R \\
\hline $\mathrm{F} 8-4 \mathrm{C}$ & 0 & 0 & 0 & 9 & 9 & 9 & 7 & 9 & 9 & 9 & 6 & PCc1-R \\
\hline F8-5A & 0 & 0 & 0 & 0 & 9 & 9 & 9 & 9 & 9 & 9 & 5 & PCc2-R \\
\hline F8-5B & 0 & 0 & 0 & 9 & 9 & 9 & 9 & 9 & 9 & 9 & 6 & PCc1-R \\
\hline SKP16 & 0 & 0 & 0 & 0 & 0 & 5 & 5 & 9 & 9 & 7 & 4 & PCc3-R \\
\hline $158 \mathrm{ci}$ & 0 & 0 & 0 & 0 & 7 & 9 & 9 & 9 & 9 & 9 & 5 & PCc2-R \\
\hline Mean & 0 & 0 & 0 & 4 & 7 & 8 & 8 & 9 & 9 & 9 & 5 & $\ldots$ \\
\hline
\end{tabular}

a Anthracnose severity scores (0 to 9 ) based on percent lesion size as a proportion to fruit size as described in Materials and Methods (pathogenicity study).

Table 4. Anthracnose severity scores on a 0 -to-9 scale and pathotypes of 12 Colletotrichum capsici isolates at the mature green fruit stage of 10 chili genotypes

\begin{tabular}{|c|c|c|c|c|c|c|c|c|c|c|c|c|}
\hline \multirow[b]{2}{*}{ Isolate } & \multicolumn{3}{|c|}{ Capsicum baccatum } & \multicolumn{2}{|c|}{ C. chinense } & \multicolumn{3}{|c|}{ C. annuum } & \multicolumn{2}{|c|}{ C. frutescens } & \multirow[b]{2}{*}{ Mean } & \multirow[b]{2}{*}{ Pathotype } \\
\hline & 80 & 81 & 1422 & 932 & 4714 & JD & BC & 83 & KN & KR & & \\
\hline F4-2C & 0 & 0 & 0 & 0 & 3 & 5 & 5 & 9 & 9 & 9 & 4 & PCc1-G \\
\hline F4-5A & 0 & 0 & 0 & 0 & 3 & 9 & 7 & 9 & 9 & 9 & 5 & PCc1-G \\
\hline F7-3A & 0 & 0 & 0 & 0 & 0 & 5 & 9 & 9 & 9 & 9 & 4 & PCc2-G \\
\hline F8-1A & 0 & 0 & 0 & 0 & 7 & 9 & 9 & 9 & 9 & 9 & 5 & PCc1-G \\
\hline F8-2A & 0 & 0 & 0 & 0 & 5 & 7 & 9 & 9 & 9 & 9 & 5 & PCc1-G \\
\hline F8-3A & 0 & 0 & 0 & 0 & 9 & 7 & 9 & 9 & 9 & 9 & 5 & PCc1-G \\
\hline F8-3B & 0 & 0 & 0 & 0 & 9 & 7 & 9 & 9 & 9 & 9 & 5 & PCc1-G \\
\hline F8-4C & 0 & 0 & 0 & 0 & 9 & 7 & 9 & 9 & 9 & 9 & 5 & PCc1-G \\
\hline F8-5A & 0 & 0 & 0 & 0 & 5 & 5 & 9 & 9 & 9 & 9 & 5 & PCc1-G \\
\hline F8-5B & 0 & 0 & 0 & 0 & 7 & 3 & 9 & 9 & 5 & 9 & 4 & PCc1-G \\
\hline SKP16 & 0 & 0 & 0 & 0 & 0 & 3 & 3 & 9 & 9 & 9 & 3 & PCc2-G \\
\hline $158 \mathrm{ci}$ & 0 & 0 & 0 & 0 & 5 & 7 & 9 & 9 & 9 & 9 & 5 & PCc1-G \\
\hline Mean & 0 & 0 & 0 & 0 & 5 & 6 & 8 & 9 & 9 & 9 & 5 & $\ldots$ \\
\hline
\end{tabular}

a Anthracnose severity scores (0 to 9 ) based on percent lesion size as a proportion to fruit size as described in Materials and Methods (pathogenicity study).

Table 5. Anthracnose severity scores on a 0-to- 9 scale $^{\mathrm{a}}$ and pathotypes of 11 Colletotrichum gloeosporioides isolates at the ripe fruit stage of 10 chili genotypes

\begin{tabular}{|c|c|c|c|c|c|c|c|c|c|c|c|c|}
\hline \multirow[b]{2}{*}{ Isolate } & \multicolumn{3}{|c|}{ Capsicum baccatum } & \multicolumn{2}{|c|}{ C. chinense } & \multicolumn{3}{|c|}{ C. annuum } & \multicolumn{2}{|c|}{ C. frutescens } & \multirow[b]{2}{*}{ Mean } & \multirow[b]{2}{*}{ Pathotype } \\
\hline & 80 & 81 & 1422 & 932 & 4714 & JD & BC & 83 & KN & KR & & \\
\hline F1-3A & 0 & 0 & 0 & 0 & 0 & 0 & 9 & 9 & 9 & 9 & 4 & PCg5-R \\
\hline F1-3C & 0 & 0 & 0 & 0 & 5 & 5 & 9 & 9 & 9 & 7 & 4 & PCg1-R \\
\hline F4-1B & 0 & 0 & 0 & 0 & 0 & 9 & 0 & 9 & 9 & 9 & 4 & PCg4-R \\
\hline F4-1C & 0 & 0 & 0 & 0 & 7 & 0 & 0 & 9 & 7 & 9 & 3 & PCg3-R \\
\hline F5-1A & 0 & 0 & 0 & 0 & 9 & 9 & 5 & 9 & 9 & 9 & 5 & PCg1-R \\
\hline F5-2D & 0 & 0 & 0 & 0 & 5 & 3 & 9 & 9 & 9 & 9 & 4 & PCg1-R \\
\hline F5-4A & 0 & 0 & 0 & 0 & 9 & 9 & 9 & 9 & 9 & 9 & 5 & PCg1-R \\
\hline F7-1B & 0 & 0 & 0 & 0 & 5 & 0 & 5 & 9 & 9 & 5 & 3 & PCg2-R \\
\hline F7-3B & 0 & 0 & 0 & 0 & 0 & 0 & 5 & 9 & 9 & 9 & 3 & PCg5-R \\
\hline F7-4A & 0 & 0 & 0 & 0 & 3 & 0 & 9 & 9 & 9 & 9 & 4 & PCg2-R \\
\hline DOA & 0 & 0 & 0 & 0 & 9 & 7 & 7 & 9 & 9 & 9 & 5 & PCg1-R \\
\hline Mean & 0 & 0 & 0 & 0 & 5 & 4 & 6 & 9 & 9 & 9 & 4 & $\ldots$ \\
\hline
\end{tabular}

a Anthracnose severity scores (0 to 9 ) based on percent lesion size as a proportion to fruit size as described in Materials and Methods (pathogenicity study). 
line C04714 and C. annuum cvs. Bangchang and Jinda. Six Colletotrichum gloeosporioides pathotypes were identified at the mature green fruit stage based on differential reactions in $\mathrm{C} 04714$ and all genotypes of Capsicum annuum and $C$. frutescens. Colletotrichum acutatum was grouped into three pathotypes at the mature green fruit stage based on differential reactions in Capsicum baccatum lines PBC80 and PBC81, while no pathotype was identified at the ripe fruit stage.

\section{DISCUSSION}

Differential host reactions that occurred in the 10 chili genotypes inoculated with three Colletotrichum spp. were a result of specific host and pathogen interactions at two fruit maturity stages.

The three pathotypes of Colletotrichum capsici at the ripe fruit stage are the same as those identified by Montri et al. (5), who used the same isolates and chili genotypes. The addition of isolate $158 \mathrm{ci}$ and screening of isolates on the additional Capsicum frutescens genotype Karen did not distinguish any new pathotypes. Isolate 158ci was identified as PCc2-R.

Previous studies $(3,4,6)$ of resistance to anthracnose in chili have shown distinct genes to be responsible for resistance at mature green and ripe fruit stages. There- fore, the different genes expressed at different fruit maturity stages reacted differentially to a Colletotrichum isolate. $C$. baccatum genotypes $\mathrm{PBC} 80$ and $\mathrm{PBC} 81$ were more susceptible to Colletotrichum acutatum isolates at the mature green fruit stage than the red fruit stage. In contrast, Capsicum chinense line PBC932 was more resistant to Colletotrichum capsici at the mature green fruit stage than the ripe fruit stage.

Among the three Colletotrichum species, Colletotrichum acutatum appeared to be the most aggressive pathogen, with the overall mean scores over 6 for both fruit maturity stages (Tables 7 and 8). C. acu-

Table 6. Anthracnose severity scores on a 0 -to- 9 scale ${ }^{\mathrm{a}}$ and pathotypes of 11 Colletotrichum gloeosporioides isolates at the mature green fruit stage of 10 chili genotypes

\begin{tabular}{|c|c|c|c|c|c|c|c|c|c|c|c|c|}
\hline \multirow[b]{2}{*}{ Isolate } & \multicolumn{3}{|c|}{ Capsicum baccatum } & \multicolumn{2}{|c|}{ C. chinense } & \multicolumn{3}{|c|}{ C. annuum } & \multicolumn{2}{|c|}{ C. frutescens } & \multirow[b]{2}{*}{ Mean } & \multirow[b]{2}{*}{ Pathotype } \\
\hline & 80 & 81 & 1422 & 932 & 4714 & JD & BC & 83 & $\mathbf{K N}$ & KR & & \\
\hline F1-3A & 0 & 0 & 0 & 0 & 0 & 0 & 0 & 0 & 9 & 0 & 1 & PCg5-G \\
\hline F1-3C & 0 & 0 & 0 & 0 & 0 & 0 & 0 & 9 & 0 & 9 & 2 & PCg4-G \\
\hline F4-1B & 0 & 0 & 0 & 0 & 0 & 0 & 0 & 9 & 9 & 9 & 3 & PCg3-G \\
\hline F4-1C & 0 & 0 & 0 & 0 & 0 & 0 & 0 & 9 & 9 & 9 & 3 & PCg3-G \\
\hline F5-1A & 0 & 0 & 0 & 0 & 0 & 0 & 0 & 9 & 9 & 7 & 3 & PCg3-G \\
\hline F5-2D & 0 & 0 & 0 & 0 & 5 & 0 & 0 & 9 & 9 & 9 & 3 & PCg1-G \\
\hline F5-4A & 0 & 0 & 0 & 0 & 0 & 0 & 0 & 7 & 7 & 9 & 2 & PCg3-G \\
\hline F7-1B & 0 & 0 & 0 & 0 & 0 & 0 & 0 & 9 & 9 & 9 & 3 & PCg3-G \\
\hline F7-3B & 0 & 0 & 0 & 0 & 0 & 0 & 0 & 0 & 0 & 7 & 1 & PCg6-G \\
\hline F7-4A & 0 & 0 & 0 & 0 & 5 & 0 & 0 & 9 & 5 & 7 & 3 & PCg1-G \\
\hline DOA & 0 & 0 & 0 & 0 & 0 & 7 & 5 & 9 & 9 & 0 & 3 & PCg2-G \\
\hline Mean & 0 & 0 & 0 & 0 & 1 & 1 & 1 & 7 & 7 & 7 & 3 & $\ldots$ \\
\hline
\end{tabular}

${ }^{a}$ Anthracnose severity scores (0 to 9 ) based on percent lesion size as a proportion to fruit size as described in Materials and Methods (pathogenicity study).

Table 7. Anthracnose severity scores on a 0-to-9 scale and pathotypes of 10 Colletotrichum acutatum isolates at the ripe fruit stage of 10 chili genotypes

\begin{tabular}{|c|c|c|c|c|c|c|c|c|c|c|c|c|}
\hline \multirow[b]{2}{*}{ Isolate } & \multicolumn{3}{|c|}{ Capsicum baccatum } & \multicolumn{2}{|c|}{ C. chinense } & \multicolumn{3}{|c|}{ C. annuum } & \multicolumn{2}{|c|}{ C. frutescens } & \multirow[b]{2}{*}{ Mean } & \multirow[b]{2}{*}{ Pathotype } \\
\hline & 80 & 81 & 1422 & 932 & 4714 & JD & BC & 83 & KN & KR & & \\
\hline 211 & 0 & 0 & 3 & 9 & 5 & 9 & 7 & 9 & 9 & 9 & 6 & $\ldots$ \\
\hline 311 & 0 & 0 & 3 & 9 & 9 & 5 & 7 & 9 & 9 & 9 & 6 & $\ldots$ \\
\hline 313 & 0 & 0 & 5 & 9 & 7 & 7 & 5 & 9 & 9 & 9 & 6 & $\ldots$ \\
\hline 314 & 0 & 0 & 5 & 9 & 7 & 9 & 7 & 9 & 9 & 9 & 6 & $\ldots$ \\
\hline 316 & 0 & 0 & 5 & 9 & 9 & 9 & 7 & 9 & 9 & 9 & 7 & $\ldots$ \\
\hline 322 & 0 & 0 & 5 & 9 & 7 & 9 & 9 & 9 & 9 & 9 & 7 & $\ldots$ \\
\hline MJ3 & 0 & 0 & 3 & 9 & 7 & 9 & 5 & 9 & 9 & 9 & 6 & $\ldots$ \\
\hline MJ5 & 0 & 0 & 7 & 9 & 9 & 7 & 5 & 9 & 9 & 9 & 6 & $\ldots$ \\
\hline MJ7 & 0 & 0 & 5 & 5 & 7 & 9 & 7 & 9 & 9 & 9 & 6 & $\ldots$ \\
\hline MJ8 & 0 & 0 & 5 & 9 & 9 & 9 & 5 & 9 & 9 & 9 & 6 & $\ldots$ \\
\hline Mean & 0 & 0 & 5 & 9 & 8 & 8 & 6 & 9 & 9 & 9 & 6 & $\ldots$ \\
\hline
\end{tabular}

${ }^{a}$ Anthracnose severity scores (0 to 9 ) based on percent lesion size as a proportion to fruit size as described in Materials and Methods (pathogenicity study).

Table 8. Anthracnose severity scores on a 0-to- 9 scale $^{\mathrm{a}}$ and pathotypes of 10 Colletotrichum acutatum isolates at the mature green fruit stage of 10 chili genotypes

\begin{tabular}{|c|c|c|c|c|c|c|c|c|c|c|c|c|}
\hline \multirow[b]{2}{*}{ Isolate } & \multicolumn{3}{|c|}{ Capsicum baccatum } & \multicolumn{2}{|c|}{ C. chinense } & \multicolumn{3}{|c|}{ C. annuum } & \multicolumn{2}{|c|}{ C. frutescens } & \multirow[b]{2}{*}{ Mean } & \multirow[b]{2}{*}{ Pathotype } \\
\hline & 80 & 81 & 1422 & 932 & 4714 & JD & BC & 83 & $\mathbf{K N}$ & KR & & \\
\hline 211 & 0 & 0 & 5 & 9 & 7 & 7 & 7 & 9 & 9 & 9 & 6 & PCa3-G \\
\hline 311 & 0 & 0 & 5 & 9 & 9 & 5 & 7 & 9 & 9 & 9 & 6 & PCa3-G \\
\hline 313 & 0 & 3 & 5 & 9 & 5 & 9 & 7 & 9 & 9 & 9 & 7 & PCa2-G \\
\hline 314 & 5 & 5 & 7 & 9 & 5 & 9 & 5 & 9 & 9 & 9 & 7 & PCa1-G \\
\hline 316 & 5 & 5 & 7 & 7 & 7 & 9 & 5 & 9 & 9 & 9 & 7 & PCa1-G \\
\hline 322 & 5 & 5 & 5 & 9 & 7 & 9 & 7 & 9 & 9 & 9 & 7 & PCa1-G \\
\hline MJ3 & 0 & 0 & 5 & 9 & 7 & 5 & 5 & 9 & 9 & 9 & 6 & PCa3-G \\
\hline MJ5 & 0 & 0 & 5 & 7 & 9 & 5 & 7 & 9 & 9 & 9 & 6 & PCa3-G \\
\hline MJ7 & 3 & 5 & 5 & 9 & 5 & 7 & 9 & 9 & 9 & 9 & 7 & PCa1-G \\
\hline MJ8 & 5 & 5 & 5 & 9 & 9 & 9 & 9 & 9 & 9 & 9 & 8 & PCa1-G \\
\hline Mean & 2 & 3 & 5 & 9 & 7 & 7 & 7 & 9 & 9 & 9 & 7 & $\ldots$ \\
\hline
\end{tabular}

${ }^{a}$ Anthracnose severity scores (0 to 9 ) based on percent lesion size as a proportion to fruit size as described in Materials and Methods (pathogenicity study). 
Table 9. Summary of numbers of pathotypes $(\mathrm{P})$ identified for the three Colletotrichum spp. at ripe $(\mathrm{R})$ and mature green $(\mathrm{G})$ fruit stages and their differential Capsicum genotypes ${ }^{\mathrm{a}}$

\begin{tabular}{|c|c|c|c|c|c|c|c|c|c|c|c|c|}
\hline \multirow[b]{2}{*}{ Colletotrichum spp. } & \multirow[b]{2}{*}{ Stage } & \multirow[b]{2}{*}{$\mathbf{P}$} & \multicolumn{3}{|c|}{ Capsicum baccatum } & \multicolumn{2}{|c|}{ C. chinense } & \multicolumn{3}{|c|}{ C. annuum } & \multicolumn{2}{|c|}{ C. frutescens } \\
\hline & & & 80 & 81 & 1422 & 932 & 4714 & JD & BC & 83 & $\mathbf{K N}$ & KR \\
\hline \multirow[t]{2}{*}{ Colletotrichum capsici } & $\mathrm{R}$ & 3 & • & • & • & \pm & \pm & + & + & + & + & + \\
\hline & $\mathrm{G}$ & 2 & • & • & • & • & \pm & + & + & + & + & + \\
\hline \multirow[t]{2}{*}{ C. gloeosporioides } & $\mathrm{R}$ & 5 & $\bullet$ & $\bullet$ & $\bullet$ & $\bullet$ & \pm & \pm & \pm & + & + & + \\
\hline & $\mathrm{G}$ & 6 & • & • & • & • & \pm & \pm & \pm & \pm & \pm & \pm \\
\hline \multirow[t]{2}{*}{ C. acutatum } & $\mathrm{R}$ & $\ldots$ & • & • & + & + & + & + & + & + & + & + \\
\hline & $\mathrm{G}$ & 3 & \pm & \pm & + & + & + & + & + & + & + & + \\
\hline
\end{tabular}

a Symbols: $-=$ no lesions incited by any isolate within a Colletotrichum spp., $+=$ lesions incited by all isolates within a Colletotrichum spp., and $\pm=$ differ ential host reactions within a Colletotrichum spp.

tatum isolates were generally able to incite lesions on all tested chili genotypes, including mature green fruit of Capsicum baccatum lines PBC80 and PBC81, which were resistant to Colletotrichum capsici and $C$. gloeosporioides. In other words, PBC80 and PBC81 contained the broadest spectrum of anthracnose resistance. $C$. capsici was the second most virulent pathogen after $C$. acutatum and more virulent than C. gloeosporioides. Capsicum chinense line PBC932, which was used as the source of anthracnose resistance to introgress into $C$. annuum elite chili $(3,6)$, was relatively resistant to Colletotrichum capsici and $C$. gloeosporioides but highly susceptible to $C$. acutatum. Of the four Capsicum spp., Capsicum frutescens cultivars and $C$. annuum line 83-168 showed the highest susceptibility to all three Colletotrichum spp.

Park et al. (8) have recently studied differential reactions of 41 Capsicum germplasm lines with 10 Korean isolates of Colletotrichum acutatum and found that only Capsicum baccatum lines were resistant. $\mathrm{PBC} 80$ and $\mathrm{PBC} 81$ were not the most resistant among the $22 \mathrm{C}$. baccatum lines tested across the Colletotrichum acutatum isolates. The study by Park et al. (8) suggested that the Capsicum baccatum germplasm pool contained higher levels of resistance to anthracnose than other Capsicum spp., which will be useful genetic resources for anthracnose resistance, and that the Korean Colletotrichum acutatum isolates may be more aggressive than the Thai isolates used in our study.

The identification of pathotypes of $C$. capsici, C. gloeosporioides, and $C$. acutatum is a significant finding that will impact on chili breeding for resistance to anthracnose, and will provide a better understanding of the specific interactions between chili genotype, Colletotrichum isolate, and chili fruit maturity.

\section{ACKNOWLEDGMENTS}

We thank the National Center for Genetic Engineering and Biotechnology, National Science and Technology Development Agency (Thailand) for financial support to the project and a Master's student scholarship; and the Center for Agricultural Biotechnology, Postgraduate Education and Research Development Office, Commission on Higher Education, Ministry of Education for partial support for this project. Field experiments were supported by the Tropical Vegetable Research Center, Kasetsart University, Kamphaeng Saen Campus, Thailand.

\section{LITERATURE CITED}

1. Kanchana-udomkan, C., Taylor, P. W. J., and Mongkolporn, O. 2004. Development of a bioassay to study anthracnose infection of chili fruit caused by Colletotrichum capsici. Thai J. of Agric. Sci. 37:293-297.

2. Kim, J. T., Park, S.-Y., Choi, W., Lee, Y.-H., and Kim, H. T. 2008. Characterization of $\mathrm{Col}-$ letotrichum isolates causing anthracnose of pepper in Korea. Plant Pathol. J. 24:17-23.

3. Mahasuk, P., Khumpeng, N., Wasee, S., Taylor, P. W. J., and Mongkolporn, O. 2009. Inheritance of resistance to anthracnose (Colletotrichum capsici) at seedling and fruiting stages in chili pepper (Capsicum spp.). Plant Breed. 128:701-706.

4. Mahasuk, P., Taylor, P. W. J., and Mongkolporn, O. 2009. Identification of two new genes conferring resistance to Colletotrichum acutatum in Capsicum baccatum L. Phytopathology 99:1100-1104.

5. Montri, P., Taylor, P. W. J., and Mongkolporn, O. 2009. Pathotypes of Colletotrichum capsici, the causal agent of chili anthracnose, in Thailand. Plant Dis. 93:17-20.

6. Pakdeevaraporn, P., Wasee, S., Taylor, P. W. J., and Mongkolporn, O. 2005. Inheritance of resistance to anthracnose caused by Colletotrichum capsici in Capsicum. Plant Breed. 124:206-208

7. Park, H. G. 2007. Problems of anthracnose in pepper and prospects for its management. Page 19 in: The First International Symposium on Chili Anthracnose, Convention Center, Seoul National University, Korea.

8. Park, S.-K., Kim, S. H., Park, H. G., and Yoon, J. B. 2009. Capsicum germplasm resistant to pepper anthracnose differentially interact with Colletotrichum isolates. Hortic. Environ. Biotechnol. 50:17-23.

9. Photita, W., Lumyong, S., Lumyong, P. McKenzie, E. H. C., Ford, R., Taylor, P. W. J. and Hyde, K. D. 2005. Morphological and molecular characterisation of Colletotrichum species from herbaceous plants in Thailand. Fungal Divers. 18:117-133.

10. Ramachandran, N., Madhavi, R. K., and Rathnamma, K. 2007. Current status of chili anthracnose in India. Page 25 in: The First Internationa Symposium on Chili Anthracnose, Convention Center, Seoul National University, Korea.

11. Than, P. P., Jeewon, R., Hyde, K. D., Pongsupasamit, S., Mongkolporn, O., and Taylor, P. W. J. 2008. Characterization and pathogenicity of Colletotrichum species associated with anthracnose on chilli (Capsicum spp.) in Thailand. Plant Pathol. 57:562-572.

12. Zhang, D., Zhu, C., and Liu, Y. 2007. Chili anthracnose research in China: an overview. Page 26 in: The First International Symposium on Chili Anthracnose, Convention Center, Seoul National University, Korea. 